\title{
Periodic orbits around triangular points in the restricted problem of three oblate bodies
}

\author{
Jagadish Singh ${ }^{1}$, Sunusi Haruna ${ }^{2}$ \\ ${ }^{1}$ Department of Mathematics, Faculty of Science, Ahmadu Bello University Zaria, Nigeria \\ ${ }^{2}$ Kano state Polytechnic, School of general studies, basic studies department, kano-Nigeria
}

Email address:

jgds2004@yahoo.com (J. Singh), siyudiso@gmail.com (S. Haruna)

\section{To cite this article:}

Jagadish Singh, Sunusi Haruna. Periodic Orbits around Triangular Points in the Restricted Problem of Three Oblate Bodies. American Journal of Astronomy and Astrophysics. Vol. 2, No. 2, 2014, pp. 22-26. doi: 10.11648/j.ajaa.20140202.12

\begin{abstract}
This paper performs a semi-analytic study of the periodic orbits around stable triangular equilibrium points when the three participating bodies are modeled as oblate spheroids, under effect of, radiation of the main masses and small change in the Coriolis and centrifugal forces. This study generalizes the one studied by AbdulRaheem and Singh, with the inclusion that the third body, due to rapid spinning, changes its shape from being a sphere, to an oblate spheroid. The orbits around these points are ellipses with long and short periodic orbits. The period, orientation, eccentricities, the semi-major and semi-minor axis of the elliptic orbits have been given. The consideration of the particle as an oblate spheroid affects all these outcomes. We clarify the discrepancies between our study and related previous studies.
\end{abstract}

Keywords: RTBP, Orbits, Oblateness, Radiation

\section{Introduction}

The restricted three-body (RTBP) has been generalized in the past years to include the case when one or both primaries are radiation source. Studies of this kind include among many, Radzievskii [8], Simmons et al [13], Singh and Leke [17]. Further generalizations are the case when the shapes of the participating bodies are not perfect spheres. Though, the shapes of the bodies in the classical RTBP are assumed to be spherical, but we find that in nature, several of these bodies are either oblate or triaxial. The Earth, Jupiter, Saturn, Ragulus, Neutron stars and black dwarfs are oblate. Some studies which have considered the non sphericity of the primaries include: SubbaRao and Sharma [23], Elipe and Ferrer [3], Sharma et al [12], Singh [15], Singh and Leke [19] and Singh [16]. Some of these cited studies have also included one or more parameters aside the bodies not being a sphere.

AbdulRaheem and Singh [1] studied the case when both primaries have the shape of an oblate spheroid and radiating, under effect of small perturbations in the Coriolis and centrifugal forces. Singh and Leke [20] modified the problem studied in AbdulRaheem and Singh [1] by assuming that, the masses of the primaries vary with time according to a law of mass variation. The same problem was extended by Singh and Haruna [22], by taking the test particle of infinitesimal mass, as an oblate spheroid. Rambaux [9] studied the rotational motion of the second largest body "Vesta" of the main asteroid belt, based on its orbital perturbations and its large triaxial shape.

Due to the rotational motion, periodic orbits exist in the RTBP, long and short periodic orbits are found around the equilibrium points. Elements that could be used to describe the motion of test particle relative to the primary and secondary bodies are categorized as orbital and non orbital elements. Angular momentum and total energy are the integrals available to measure the shapes and sizes of the orbits but are not directly observable. Therefore, eccentricities, inclination and semi major axes of the orbits are used to determine the shapes, orientation and sizes of the orbits.

Szebehely [24] gave a complete result regarding the periodic motion of planar circular RTBP. Sharma [11] examined the linear stability of the restricted problem in which the larger primary body is a source of radiation and the smaller one is an oblate spheroid. The eccentricity of the retrograde elliptical periodic orbits was shown to increase with oblateness and decrease with radiation force. By using second order parametric expansions, the families of periodic orbits generated from the inner collinear equilibrium point in a binary system were numerically determined by Ragos et al. [10]. Perdios [5] studied critical 
symmetric periodic orbits in the photogravitational RTBP in which the first primary is a source of radiation. Later, Perdios and Kalantonis [6] modified the problem by considering the first primary to be an oblate spheroid. The combine effect of radiation, perturbations and oblateness on the periodic orbits was carried out by AbdulRaheem and Singh [2]. Singh and Begha [18] looked at the problem of the periodic orbits when the larger and smaller primaries are triaxial and oblate bodies respectively under effects of small perturbations in the Coriolis and centrifugal forces. Perdiou et al [7] studied the periodic motions in the spatial Chermnykh's restricted three-body problem. Kishor and Kushvah [4] considered the periodic orbits in the generalized photogravitational Chermnykh-like problem with power law profile, while Singh and Leke [21] examined the periodic orbits of an axisymmetric particle under the gravitational forces of a radiating bigger primary and an oblate smaller one when they are surrounded by a belt.

Because of the ambiguity of the calculations involved, when dealing with up to five parameters of the system, we observe that some of these studies are mathematically flawed.

In this paper, we review and then extend the paper by AbdulRaheem and Singh [2] by assuming that the third body is modeled as an oblate spheroid. The paper organization is as follows; section 2 and 3 highlight the equations of motion, location of the triangular points and the periodic orbits, respectively. In sections 4, we discuss the elliptic orbits, its orientation, eccentricities and axes, and we conclude the paper in section 5 .

\section{Equations of Motion and Triangular Equilibrium Points}

Let $m_{1}$ and $m_{2}$ be the masses of the primary and secondary stars, respectively, and $m$ be the mass of the third body. Using dimensionless variables, the equations of motion of the third body in a barycentric rotating coordinate system, can be written (Singh and Haruna [22]) as

$$
\begin{gathered}
\ddot{x}-2 \phi n \dot{y}=\Omega_{x}, \ddot{y}+2 \phi n \dot{x}=\Omega_{y}, \ddot{z}=\Omega_{z} \\
\Omega=\frac{n^{2} \psi}{2}\left[(1-\mu) r_{1}^{2}+\mu r_{2}^{2}\right]+\frac{q_{1}(1-\mu)}{r_{1}}+\frac{q_{2} \mu}{r_{2}}+\frac{q_{1} A_{1}(1-\mu)}{2 r_{1}^{3}}+\frac{q_{2} A_{2} \mu}{2 r_{2}^{3}}+\frac{A_{3}(1-\mu)}{2 r_{1}^{3}}+\frac{A_{3} \mu}{2 r_{2}^{3}}
\end{gathered}
$$

where $n^{2}=1+\frac{3}{2} A_{1}+\frac{3}{2} A_{2}$

$$
r_{1}^{2}=(x-\mu)^{2}+y^{2}+z^{2}, r_{2}^{2}=(x-\mu+1)^{2}+y^{2}+z^{2}
$$

$\mu$ is the mass parameter, $n$ is the mean motion of the primaries; $r_{1}$ and $r_{2}$ are distances of the test particle from the primaries. $A_{i}(i=1,2,3)$ are oblateness coefficients of the three bodies respectively and are assumed to be very small. $R$ is the dimensional distance between them. $q_{1}$ is the radiation factor of the larger primary and given by the expression $F_{p i}=F_{g i}\left(1-q_{i}\right): 0<\left(1-q_{i}\right)<<1$ where $F_{g i}$ and $F_{p i}$ are respectively the gravitational and radiation pressure forces. $\phi=1+\epsilon ; \in<<1, \psi=1+\epsilon^{\prime} ; \epsilon^{\prime}<<1$ are parameters for the Coriolis and centrifugal forces respectively to which small perturbations $\in$ and $\epsilon^{\prime}$ are given.

Equations (1) admits the Jacobi integral

$$
C=2 \Omega-\left(\dot{x}^{2}+\dot{y}^{2}\right)
$$

where $C$ is the Jacobi constant.

Now, the triangular equilibrium points are given (Singh and Haruna [22]):

$$
\begin{aligned}
& x=\mu-\frac{1}{2}+\psi^{-2 / 3}\left[\frac{1}{3}\left(1-q_{1}\right)-\frac{1}{3}\left(1-q_{2}\right)-\frac{1}{2} \psi^{2 / 3}\left(A_{1}-A_{2}\right)\right] \\
& y= \pm \frac{\sqrt{4-\psi^{2 / 3}}}{2 \psi^{1 / 3}}\left[1-\frac{2}{4-\psi^{2 / 3}}\left\{\frac{1}{3}\left(1-q_{1}\right)+\frac{1}{3}\left(1-q_{2}\right)+\left(A_{1}+A_{2}\right)-\frac{\psi^{2 / 3}}{2}\left(A_{1}+A_{2}+2 A_{3}\right)\right\}\right]
\end{aligned}
$$

The characteristic equation and its roots are given, respectively, as (Singh and Haruna [22]),

$$
\lambda^{4}-a \lambda^{2}+b=0
$$

where

$$
\begin{gathered}
b=3 \epsilon^{\prime}-4-8 \in-6\left(A_{1}+A_{2}\right)+3\left\{1+\frac{5}{2} A_{1}+\frac{5}{2} A_{2}+A_{3}-\mu\left(A_{1}-A_{2}\right)\right\} \\
c=\frac{3}{4} \mu(1-\mu)\left[9+39\left(A_{1}+A_{2}\right)+12 A_{3}+2\left(1-q_{1}\right)+2\left(1-q_{2}\right)+2 \epsilon^{\prime}\right]
\end{gathered}
$$

and

$$
\lambda_{1,2}= \pm s_{1}, \lambda_{3,4}= \pm s_{2}
$$

where $s_{1,2}^{2}=\frac{-b \pm \sqrt{\Delta}}{2}, \Delta=b^{2}-4 c$

\section{Periodic Orbits}

In the stable region, the roots (5) are pure imaginary roots and so the motion is bounded. In this case, we have two harmonic motions, given by

$$
\begin{aligned}
& \xi=C_{1} \cos s_{1} t+S_{1} \sin s_{1} t+C_{2} \cos s_{2} t+S_{2} \sin s_{2} t \\
& \eta=\bar{C}_{1} \cos s_{1} t+\bar{S}_{1} \sin s_{1} t+\bar{C}_{2} \cos s_{2} t+\bar{S}_{2} \sin s_{2} t
\end{aligned}
$$

where

$$
\begin{aligned}
s_{1}= & {\left[\mu(1-\mu)\left\{\frac{27}{4}+\frac{117}{4}\left(A_{1}+A_{2}\right)+9 A_{3}+\frac{3}{2}\left(1-q_{1}\right)+\frac{3}{2}\left(1-q_{2}\right)+\frac{33 \epsilon^{\prime}}{2}\right\}\right]^{\frac{1}{2}} } \\
s_{2}= & {\left[1-3 \epsilon^{\prime}+8 \epsilon-\left(\frac{3}{2}+\frac{105}{4} \mu-\frac{117}{4} \mu^{2}\right) A_{1}+\left(\frac{3}{2}-\frac{129}{4} \mu+\frac{117}{4} \mu^{2}\right) A_{2}-3\left(1-3 \mu+3 \mu^{2}\right) A_{3}\right.} \\
& \left.-\frac{27}{4} \mu(1-\mu)-\frac{3}{2}\left(1-q_{1}\right) \mu(1-\mu)-\frac{3}{2}\left(1-q_{2}\right) \mu(1-\mu)-\frac{33 \epsilon^{\prime}}{2} \mu(1-\mu)\right]^{\frac{1}{2}}
\end{aligned}
$$


$S_{1}$ is the frequency of the long periodic orbits while $S_{2}$ is for the short periodic orbits. The coefficients; $C_{1}, S_{1}, \bar{C}_{1}, \bar{S}_{1}$ and $C_{2}, S_{2}, \bar{C}_{2}, \bar{S}_{2}$ are the long and short periodic terms, respectively.

\section{Elliptic Orbits}

Now, the expansion of the force function $\Omega$ around the triangular points $(x, y)$ is expressed as

$$
\begin{aligned}
\Omega=\Omega^{0}+\Omega_{x x}^{0}\left(\frac{\xi^{2}}{2}\right)+\Omega_{y y}^{0}\left(\frac{\eta^{2}}{2}\right)+\Omega_{x y}^{0} \xi \eta+o\left(\xi^{3}, \eta^{3}\right) \\
B_{1}=\frac{3}{8}+\left(\frac{27}{16}-\frac{3}{2} \mu\right) A_{1}+\left(\frac{3}{16}+\frac{3}{2} \mu\right) A_{2}-\left(\frac{1}{4}-\frac{3}{4} \mu\right)\left(1-q_{1}\right)+\left(\frac{1}{2}-\frac{3}{4} \mu\right)\left(1-q_{2}\right)+\frac{5}{8} \epsilon^{\prime} \\
B_{2}=\sqrt{3}\left[\frac{3}{4}-\frac{3}{2} \mu+\left(\frac{19}{8}-\frac{13}{4} \mu\right) A_{1}+\left(\frac{7}{8}-\frac{13}{4} \mu\right) A_{2}+\left(\frac{1}{2}+\mu\right) A_{3}-\left(\frac{1}{6}+\frac{1}{6} \mu\right)\left(1-q_{1}\right)+\left(\frac{1}{3}+\frac{1}{6} \mu\right)\left(1-q_{2}\right)+\left(\frac{11}{12}-\frac{11}{6} \mu\right) \epsilon^{\prime}\right]
\end{aligned}
$$

Equation (8) is in quadratic form, and shows that the periodic orbits around the triangular points are elliptical.

\subsection{Orientation}

If we express equation (8) as

$$
\Omega=B_{1} \xi^{2}-B_{2} \xi \eta+B_{3} \eta^{2}+B_{4}
$$

where

$$
\begin{aligned}
& B_{3}=\frac{9}{8}+\frac{33}{16}\left(A_{1}+A_{2}\right)+\frac{3}{2} A_{3}+\left(\frac{1}{4}-\frac{3}{4} \mu\right)\left(1-q_{1}\right)-\left(\frac{1}{2}-\frac{3}{4} \mu\right)\left(1-q_{2}\right)+\frac{7}{8} \epsilon^{\prime} \\
& B_{4}=\frac{3}{2}+\left(\frac{5}{4}-\frac{1}{2} \mu\right) A_{1}+\left(\frac{3}{4}+\frac{1}{2} \mu\right) A_{2}+\frac{1}{2} A_{3}-(1-\mu)\left(1-q_{1}\right)-\mu\left(1-q_{2}\right)+\frac{1}{2} \epsilon^{\prime}
\end{aligned}
$$

We introduce the variables $\bar{\xi}$ and $\bar{\eta}$ by the transformation

$$
\begin{aligned}
& \xi=\bar{\xi} \cos \alpha-\bar{\eta} \sin \alpha \\
& \eta=\bar{\xi} \sin \alpha+\bar{\eta} \cos \alpha \\
& +\left[\frac{5}{8}+\frac{1}{4} \sin ^{2} \alpha-\frac{11 \sqrt{3}(1-2 \mu)}{24} \sin 2 \alpha\right] \epsilon^{\prime} \\
& +\left[\frac{5}{8}+\frac{1}{4} \cos ^{2} \alpha+\frac{11(1-2 \mu) \sqrt{3}}{24} \sin 2 \alpha\right] \epsilon^{\prime} \\
& \bar{B}_{3}=B_{4} \\
& \tan 2 \alpha=\sqrt{3}\left[1-2 \mu+\left(\frac{8}{3}-\frac{16}{3} \mu+4 \mu^{2}\right) A_{1}-\left(\frac{4}{3}-\frac{8}{3} \mu+4 \mu^{2}\right) A_{2}-\left(\frac{4}{3}-\frac{16}{3} \mu\right) A_{3}\right. \\
& \left.+\left(\frac{8}{9}-\frac{28 \mu}{9}+4 \mu^{2}\right)\left(1-q_{1}\right)+\left(\frac{16}{9}-\frac{44}{9} \mu+4 \mu^{2}\right)\left(1-q_{2}\right)+\left(\frac{8}{9}-\frac{16}{9} \mu\right) \epsilon^{\prime}\right]
\end{aligned}
$$$$
\bar{B}_{1}=\frac{3}{8}+\frac{3}{4} \sin ^{2} \alpha-\frac{3 \sqrt{3}}{8}(1-2 \mu) \sin 2 \alpha+\left[\frac{27}{16}-\frac{3}{2} \mu+\left(\frac{3}{8}+\frac{3}{2} \mu\right) \sin ^{2} \alpha-\frac{\sqrt{3}}{2}\left(\frac{19}{8}-\frac{13}{4} \mu\right) \sin 2 \alpha\right] A_{1}
$$$$
+\left[\frac{3}{16}+\frac{3}{2} \mu+\left(\frac{15}{8}-\frac{3}{2} \mu\right) \sin ^{2} \alpha-\frac{\sqrt{3}}{2}\left(\frac{7}{8}-\frac{13}{4} \mu\right) \sin 2 \alpha\right] A_{2}+\left[\frac{3}{2} \sin ^{2} \alpha-\frac{\sqrt{3}}{2}\left(\frac{1}{2}+\mu\right) \sin 2 \alpha\right] A_{3}
$$$$
+\left[\left(-\frac{1}{4}+\frac{3}{4} \mu\right) \cos 2 \alpha+\frac{\sqrt{3}}{2}\left(\frac{1}{6}+\frac{1}{6} \mu\right) \sin 2 \alpha\right]\left(1-q_{1}\right)+\left[\left(\frac{1}{2}-\frac{3}{4} \mu\right) \cos 2 \alpha+\frac{\sqrt{3}}{2}\left(-\frac{1}{3}+\frac{1}{6} \mu\right) \sin 2 \alpha\right]\left(1-q_{2}\right)
$$$$
\bar{B}_{2}=\frac{3}{8}+\frac{3}{4} \cos ^{2} \alpha+\frac{3 \sqrt{3}}{8}(1-2 \mu) \sin 2 \alpha+\left[\frac{27}{16}-\frac{3}{2} \mu+\left(\frac{3}{8}+\frac{3}{4} \mu\right) \cos ^{2} \alpha+\frac{\sqrt{3}}{2}\left(\frac{19}{8}-\frac{13}{4} \mu\right) \sin 2 \alpha\right] A_{1}
$$$$
+\left[\frac{3}{16}+\frac{3}{2} \mu+\left(\frac{15}{8}-\frac{3}{2} \mu\right) \cos ^{2} \alpha+\frac{\sqrt{3}}{2}\left(\frac{7}{8}-\frac{13}{4} \mu\right) \sin 2 \alpha\right] A_{2}+\left[\frac{3}{2} \cos ^{2} \alpha+\frac{\sqrt{3}}{2}\left(\frac{1}{2}+\mu\right) \sin 2 \alpha\right] A_{3}
$$$$
+\left[\left(\frac{1}{4}-\frac{3}{4} \mu\right) \cos 2 \alpha-\frac{\sqrt{3}}{2}\left(\frac{1}{6}+\frac{1}{6} \mu\right) \sin 2 \alpha\right]\left(1-q_{1}\right)+\left[\left(-\frac{1}{2}+\frac{3 \mu}{4}\right) \cos 2 \alpha+\frac{\sqrt{3}}{2}\left(\frac{1}{3}-\frac{\mu}{6}\right) \sin 2 \alpha\right]\left(1-q_{2}\right)
$$

\subsection{Eccentricities of the Ellipses}

Now, the corresponding characteristics equation of the Jacobi integral $C=2 \Omega$ is quadratic has the form:

Where

This is equivalent to the rotation of the coordinate system $\xi$ and $\eta$ through angle $\alpha$. Therefore, we select $\alpha$ such that the terms containing $\bar{\xi} \bar{\eta}$ in the force function $\Omega$ vanishes. The new force function in its

$$
\Omega=\bar{B}_{1} \bar{\xi}^{2}+\bar{B}_{2} \bar{\eta}^{2}+\bar{B}_{3}
$$


The eccentricities are given (Szebehely [24]) by equations

$$
e_{i}=\left(1-\delta_{i}^{2}\right)^{1 / 2}
$$

$$
\delta_{1}^{2}=3 \mu-12 \mu^{2}-2\left(\mu+35 \mu^{2}\right) A_{1}+4\left(\mu-22 \mu^{2}\right) A_{2}-2\left(\mu+11 \mu^{2}\right) A_{3}+\frac{2}{3} \mu(1-\mu)\left(1-q_{1}\right)+\frac{2}{3} \mu(1-\mu)\left(1-q_{2}\right)+\frac{4}{3} \mu(1-34 \mu) \epsilon^{\prime}
$$

and

$$
\begin{aligned}
& \delta_{2}^{2}=\frac{1}{4}-\frac{9}{16} \mu\left(1-\frac{25 \mu}{2}\right)+\left(\frac{9}{8}-\frac{27 \mu}{16}+\frac{507 \mu^{2}}{8}\right) A_{1}-\left(\frac{3}{8}-\frac{57 \mu}{16}+\frac{294 \mu^{2}}{4}\right) A_{2}-\left(\frac{3}{4}+\frac{15 \mu}{8}-\frac{273 \mu^{2}}{16}\right) A_{3} \\
& -\left(\frac{1}{8} \mu+\frac{13 \mu^{2}}{4}\right)\left(1-q_{1}\right)-\left(\frac{1}{8} \mu+\frac{13 \mu^{2}}{4}\right)\left(1-q_{2}\right)+\left(1+\frac{63 \mu}{4}-\frac{63 \mu^{2}}{4}\right) \in-\left(\frac{3}{4}+\frac{49 \mu}{8}+\frac{695 \mu^{2}}{32}\right) \epsilon^{\prime}
\end{aligned}
$$

Hence, the eccentricity of the long and short period orbit

$$
\begin{aligned}
& e_{1}=1-\frac{3}{2} \mu+6 \mu^{2}+\left(\mu+35 \mu^{2}\right) A_{1}-2\left(\mu-22 \mu^{2}\right) A_{2}+\left(\mu+11 \mu^{2}\right) A_{3}-\frac{1}{3} \mu(1-\mu)\left(1-q_{1}\right) \\
& -\frac{1}{3} \mu(1-\mu)\left(1-q_{2}\right)-\frac{2}{3} \mu(1-34 \mu) \epsilon^{\prime} \\
& e_{2}=\frac{\sqrt{3}}{2}\left[1+\frac{3}{8} \mu(1-\mu)+\frac{81}{16} \mu^{2}+\left(\frac{3}{4}-\frac{9}{8} \mu+\frac{169}{4} \mu^{2}\right) A_{1}+\left(\frac{1}{4}-\frac{19}{8} \mu+49 \mu^{2}\right) A_{2}+\left(\frac{1}{2}+\frac{5}{4} \mu-\frac{91}{8} \mu^{2}\right) A_{3}\right. \\
& \left.+\left(\frac{1}{12} \mu+\frac{13 \mu^{2}}{2}\right)\left(1-q_{1}\right)+\left(\frac{1}{12} \mu+\frac{13 \mu^{2}}{2}\right)\left(1-q_{2}\right)-\left(\frac{2}{3}+\frac{21 \mu}{2}-\frac{21 \mu^{2}}{2}\right) \in+\left(\frac{1}{2}+\frac{49}{3} \mu+\frac{695 \mu^{2}}{12}\right) \epsilon^{\prime}\right]
\end{aligned}
$$

\subsection{Semi-Major and Semi-Minor Axes}

The lengths of the semi-major axis, $a_{i}$ and the semiminor axis, $b_{i}$ of the long and short period are obtained from the respective relations below

$$
a_{i}=\frac{1}{\delta_{i}}\left(\xi_{0}^{2} \delta_{i}^{2}+\eta_{0}^{2}\right)^{1 / 2}, b_{i}=\left(\xi_{0}^{2} \delta_{i}^{2}+\eta_{0}^{2}\right)^{1 / 2}(i=1,2)
$$

where $\xi_{0}, \eta_{0}$ are given in (3) as initial conditions (Szebehely[24]).

Then,

$$
\begin{array}{r}
\xi_{0}^{2}=\frac{1}{4}\left[1-4 \mu+4 \mu^{2}+(2-4 \mu) A_{1}+(-2+4 \mu) A_{2}+\left(-\frac{4}{3}+\frac{8}{3} \mu\right)\left(1-q_{1}\right)+\left(\frac{4}{3}-\frac{8}{3} \mu\right)\left(1-q_{2}\right)\right] \\
\eta_{0}^{2}=\frac{3}{4}\left[1-\frac{2}{3}\left(A_{1}+A_{2}\right)+\frac{4}{3} A_{3}-\frac{4}{9}\left(1-q_{1}\right)-\frac{4}{9}\left(1-q_{2}\right)-\frac{8}{9} \epsilon^{\prime}\right]
\end{array}
$$

Substituting $\chi_{i}^{2}(i=1,2)$ and equations (20) in equations (19), we get the following equations respectively

$$
\begin{aligned}
& a_{1}=\frac{\sqrt{5}}{2}\left[1+\frac{1}{10 \mu}-\frac{2}{5} \mu(1-\mu)+\left(\frac{34}{15}-\frac{2}{5} \mu\right) A_{1}+\left(\frac{37}{15}-\frac{1}{5 \mu}+\frac{2}{5} \mu\right) A_{2}+\left(\frac{19}{15}+\frac{1}{5 \mu}\right) A_{3}\right. \\
& \left.-\left(\frac{13}{45}+\frac{1}{5 \mu}-\frac{4}{15} \mu\right)\left(1-q_{1}\right)-\left(\frac{1}{45}+\frac{4}{15} \mu+\frac{1}{15 \mu}\right)\left(1-q_{2}\right)-\left(\frac{2}{9}+\frac{2}{15 \mu}\right) \epsilon^{\prime}\right] \\
& a_{2}=\frac{\sqrt{13}}{2}\left[1+\frac{23}{26} \mu+\frac{683 \mu^{2}}{52}+\left(\frac{24}{13}-\frac{103}{26} \mu-\frac{2817}{26} \mu^{2}\right) A_{1}+\left(\frac{4}{13}-\frac{185}{26} \mu-\frac{3303}{26} \mu^{2}\right) A_{2}+\right. \\
& \left(2+\frac{63}{13} \mu-\frac{162}{13} \mu^{2}\right) A_{3}-\left(\frac{10}{39}+\frac{5}{39} \mu-\frac{3}{13} \mu^{2}\right)\left(1-q_{1}\right)-\left(\frac{2}{13}+\frac{1}{3} \mu-\frac{3}{13} \mu^{2}\right)\left(1-q_{2}\right) \\
& \left.-\left(\frac{24}{13}-\frac{378 \mu}{13}-\frac{378 \mu^{2}}{13}\right) \epsilon+\left(\frac{114}{117}-\frac{102 \mu}{13}+\frac{627 \mu^{2}}{13}\right) \epsilon^{\prime}\right]
\end{aligned}
$$

$$
\begin{aligned}
& b_{1}=\frac{\sqrt{3}}{2}\left[1+\frac{1}{2} \mu-4 \mu^{2}+\left(-\frac{1}{3}+\frac{2}{3} \mu-\frac{49}{3} \mu^{2}\right) A_{1}-\left(\frac{1}{3}+\frac{1}{3} \mu+\frac{34}{3} \mu^{2}\right) A_{2}+\left(\frac{2}{3}-\frac{1}{3} \mu-\frac{7}{3} \mu^{2}\right) A_{3}\right. \\
& \left.-\left(\frac{2}{9}+\frac{5}{9} \mu-\frac{31}{9} \mu^{2}\right)\left(1-q_{1}\right)-\left(\frac{2}{9}+\frac{7}{9} \mu-\frac{41}{9} \mu^{2}\right)\left(1-q_{2}\right)+\left(-\frac{4}{9}+\frac{2}{9} \mu-\frac{76}{9} \mu^{2}\right) \epsilon^{\prime}\right]
\end{aligned}
$$

$b_{2}=\frac{\sqrt{13}}{4}\left[1-\frac{25}{104} \mu-\frac{121}{208} \mu^{2}+\left(-\frac{21}{52}+\frac{65}{104} \mu-\frac{1374}{104} \mu^{2}\right) A_{1}+\left(-\frac{23}{52}+\frac{115}{104} \mu-\frac{1239}{64} \mu^{2}\right) A_{2}+\right.$

$\left(\frac{1}{2}+\frac{9}{52} \mu+\frac{345}{52} \mu^{2}\right) A_{3}+\left(-\frac{10}{39}+\frac{31}{156} \mu-\frac{10}{13} \mu^{2}\right)\left(1-q_{1}\right)-\left(\frac{2}{13}+\frac{37}{156} \mu+\frac{1}{13} \mu^{2}\right)\left(1-q_{2}\right)$

$\left.+\left(\frac{8}{13}-\frac{158}{13} \mu+\frac{410}{13} \mu^{2}\right) \in-\left(\frac{41}{78}+\frac{9}{26} \mu+\frac{367}{52} \mu^{2}\right) \epsilon^{\prime}\right]$

\section{Discussion and Conclusion}

We have generalized the study of the periodic orbits of the model studied by AbdulRaheem and Singh [2], when the test particle is an oblate spheroid. This paper is also the study of the periodic orbits of the triangular points studied by Singh and Haruna [22]. Clearly, the equations of motion of our studies and those of AbdulRaheem and Singh [2] differ only due to oblateness of the test particle in the force function (2). Also, the positions of the triangular equilibrium points differ only because of the shape of the test particle. These equations become the same when the test particle is a sphere

Now, equations (7) and (8) are the frequencies of the long and short period orbits, respectively. They differ from those in AbdulRaheem and Singh [2], because of the inclusion that the test particle is an oblate spheroid. But, we observe that the coefficients of the oblateness of the bigger primary are both incorrect. The coefficients of oblateness of the test particle worked out by us are same with those of Singh and Leke [21]. When the bodies are spherical, non radiating and no small change in the Coriolis and centrifugal forces, we have $s_{1}=\frac{3 \sqrt{3 \mu}}{2}$ and $s_{2}=1-\frac{27}{8} \mu$; showing 
that $s_{2}>s_{1}$.

We see from equation (9) that the orbits are elliptical while equation (10), give the orientation of the orbit. We note that the first coefficient in the equation are same with that in AbdulRaheem and Singh [2], except for what seems like a typographic error in the coefficient of the oblateness of the bigger primary. However, the coefficients of the term containing triaxiality of the bigger primary in the paper by Singh and Begha [18] are equally erroneous. In the second coefficient of (10), the term containing a small change in the centrifugal in AbdulRaheem and Singh [2] are different while the other terms are same with ours. In Singh and Begha [18], the all the terms there are a found in ours when $\sigma_{1}=\sigma_{2}$. In the third coefficient, our results are in accordance with that in AbdulRaheem and Singh [2] but again the term containing the small change, differs. We observe that, the second, third and fourth coefficients in equation (10) contain an additional term containing oblateness of the test particle. Our results in equation (10) fully agree with the corresponding terms in those of Singh and Leke [21]. The same disparities are also observed in equations (12).

Equation (13) demonstrates that the orientation of these orbits may increase. This will depend on the parameters of the system. If an increase or decreases occur, then this will produce a change in the orientation of the orbits along the $\bar{\xi}$ coordinate. Some differences are also noticed in the eccentricities of the long and short periodic orbits which are given in equations (18), with those in, AbdulRaheem and Singh [2], and Singh and Begha [18]. Though, besides the oblateness of the test particle, the values in ours and those in AbdulRaheem and Singh [2], depend on the mass ratio, radiation pressure forces, oblateness of the primaries, and small perturbations. The same thing can be said about the semi-major and semi-minor axes of the long and short period orbit given in equations (21) to (24). They are affected by oblateness of the test particle.

Finally, we have followed the same pattern as previous studies and have in particular retained the first term of the product $\mu^{2}(1-\mu)$ and $\mu^{2}(1-\mu)^{2}$ done in Singh and Leke [21].

\section{References}

[1] AbdulRaheem, A., Singh, J.: AJ, 131, 1880 (2006)
[2] AbdulRaheem, A., Singh, J.: Astrophys. Space Sci. 317, 9 (2008)

[3] Elipe, A., \& Ferrer, S. Celest. Mech., 37, 59 (1985)

[4] Kishor, R., Kushvah, B.S.: Astrophys. Space Sci. 334, 333(2013)

[5] Perdios, E. A.: Astrophys. Space Sci., 286, 501(2003)

[6] Perdios, E. A., Kalantonis, V.S.: Astrophys. Space Sci., 305, 331(2006),

[7] Perdiou, A.E., Nikaki, A.A., Perdios, E.A.: Astrophys. Space Sci., 345, 57(2013)

[8] Radzievskii, V.V.: Astron. J. 27, 250(1950)

[9] Rambaux, N.: Astron. \& Astrophys., 556, 151 (2013).

[10] Ragos, O., Zagouras, C. G., Perdios, E.: Astrophys. Space Sci. 182, 313 (1991)

[11] Sharma, R.K.: Astrophys. Space Sci. 185, 271 (1987)

[12] Sharma, R.K., Taqvi, Z.A., Bhatnagar, K. B.: Celest. Mech. Dyn. Astron. 79, 119 (2001).

[13] Simmons, J.F.L., McDonald, J.C., Brown, J.C.: Celest. Mech. 35, 145 (1985)

[14] Singh, J.: Astrophys. Space Sci., 342, 303(2012)

[15] Singh, J.: AJ. 137, 3286 (2009)

[16] Singh, J.: Astrophys. Space Sci., 346, 41(2013)

[17] Singh, J., Leke, O.: Astrophys. Space Sci., 326, 305(2010)

[18] Singh, J., Begha, J. M.: Astrophys. Space Sci., 331, 511(2011)

[19] Singh, J., Leke, O.: Astrophys. Space Sci., 340, 27(2012)

[20] Singh, J., Leke, O.: Astrophys. Space Sci., 344, 51(2013)

[21] Singh, J., Leke, O.: Astrophys. Space Sci. 10.1007/s10509013-1702-0 (2014)

[22] Singh, J., Haruna, S.: Astrophys. Space Sci., 349, 107 (2014)

[23] SubbaRao, P.V., Sharma, R. k.: Astron. \& Astrophys.43, 381(1975)

[24] Szebehely, V.G.: Theory of Orbits, Academic press, New York (1967) 